\title{
Role of potassium fertilizer in improving yield and its components for some sesame varieties under salt-affected soil conditions
}

\author{
Abdelsatar, M.A. ${ }^{1 *}$, Huda M.M. Elmasry ${ }^{2}$ and M.A. Attia ${ }^{1}$ \\ ${ }^{1}$ Oil Crops Research Department, Field Crops Research Institute, Agricultural Research Center, Giza, Egypt. \\ ${ }^{2}$ Soil, Water and Environment Research Institute, Agriculture Research Center, Giza, Egypt.
}

\begin{abstract}
Improving yield and its components for some sesame genotypes using potassium fertilizer application under salt-affected soil was the desired goal of this study. So, for each site i.e. non-saline site, moderately-saline site and highly-saline site, the experiment was performed in a randomized complete block design (RCBD) using split-plot arrangement with three replications at the Investigational Farm of Tag-El-Ezz Agricultural Research Station, Dakahlia Governorate, Agricultural Research Center, Egypt during 2018 and 2019 successive summer seasons. Three levels of potassium fertilizer i.e. 0, 25 and $50 \mathrm{~kg} \mathrm{~K}_{2} \mathrm{O}$ fed $^{-1}$ were assigned in the main plots and three sesame varieties i.e. Shandaweel 3, Giza 32 and Sohag 1 were arranged in the sub-plots. The main effects of sites, potassium fertilizer levels, sesame varieties and their dual and triple interactions were highly significant for all the studied traits. Shandaweel 3 was more salt tolerant variety and it was relevant to grow under salt stress. The potassium rate of $50 \mathrm{~kg} \mathrm{~K}_{2} \mathrm{O} \mathrm{fed}^{-1}$ was found to be more efficient to mitigate salinity effect and increase yield and its components under salt stress. Therefore, Shandaweel 3 could be recommended under salinity stress with adding $50 \mathrm{~kg} \mathrm{~K}_{2} \mathrm{O} \mathrm{fed}{ }^{-1}$ to get high yield with high quality and nutrition value.
\end{abstract}

Keywords: Potassium, Salt-affected soils, Seed chemical composition contents, Varieties, Yield

\section{Introduction}

Sesame is one of the most essential oil crops in Egypt and the world due to its seeds which had high oil content and high nutritional value compared to other oil crops. The population increase is obliged us to cultivate at salt-affected soil, the sesame crop is one of the targeted crops to expand its cultivation at these soils. Soil salinity is one of the adverse edaphic conditions that limits sesame yield. In this regard, study of Ali et al. (2005), Gaballah et al. (2007), AbdelRahman (2014), Nóbrega et al. (2018) and

\footnotetext{
*Corresponding author: Mohamed Ali Abdelsatar E-mail:mohmedtemraz1@yahoo.com

Received: January 9, 2021;

Accepted: January 28, 2021;

Published: January 30, 2021.
}

Vadaliya et al. (2019) found that progressively decreased in plant height, leaf area, number of branches per plant, total dry matter, number of capsule per plant, number of seeds per capsule, 1000-seed weight, seed weight per plant, and harvest index associated with increasing salinity levels. Application of potassium fertilizer is one of methods to improve the performance of plants under salt-affected soil conditions, as it had a vital role in regulating the vital processes in the plant and increases its ability to tolerance salt stress as well as its role in assimilate translocation from source to sink. In this regard, potassium fertilization had a major role in increasing yield and its components, according to a study of Hafiz and El-Bramawy (2012), 
Abdel-Rahman (2014), Jat et al. (2017) and Ahmad et al. (2018).

The genetic potential of varieties has the greatest effect on salinity tolerance with increasing productivity under salt-affected soil conditions. In this regard, some sesame genotypes had the largest effect on increasing productivity under salt-affected soil conditions, as is evident in the study of Gaballah et al. (2007), Suassuna et al. (2017) and Vadaliya et al. (2019). As previously mentioned, the previous studies provided us with the facts about the effective role of potassium fertilizer application and genetic potential for increasing yield and its components under saltaffected soil conditions.

Therefore, this research aimed to find out the role of potassium fertilizer and genetic potential in reducing effect of salt-affected soil on yield and its components as well as their effects on chemical composition contents of sesame seeds.

\section{Materials and Methods \\ Site description}

Field trials were performed under three saltaffected sites i.e. non-saline site, moderatelysaline site and highly-saline site at the Experimental Farm of Tag-El-Ezz Agricultural Research Station, Dakahlia Governorate, Agricultural Research Center in Egypt $\left(31^{\circ} 36^{\prime}\right.$, $30^{\circ} \quad 57^{\prime}$ ) during the two successive summer seasons of 2018 and 2019. Soil samples (0-30 $\mathrm{cm})$ collected from the three saline sites and analyzed for physical and chemical characteristics as suggested by Jackson (1973) as in Table 1. The earlier crop in both seasons was wheat.

Table 1. Physicals and chemicals analyses of experimental sites at $0-30 \mathrm{~cm}$ depth of soil.

\begin{tabular}{|c|c|c|c|c|c|c|c|c|c|c|c|}
\hline \multicolumn{12}{|c|}{ Pre-sowing } \\
\hline \multirow{2}{*}{ Sites } & \multirow{2}{*}{ Seasons } & \multicolumn{3}{|c|}{ Available PPM } & \multirow{2}{*}{ pH } & \multirow{2}{*}{$\begin{array}{c}\mathrm{EC} \\
(\mathrm{mmh} / \mathrm{cm})\end{array}$} & \multirow{2}{*}{ Sites category } & \multirow{2}{*}{ Clay \% } & \multirow{2}{*}{$\begin{array}{c}\text { Silt } \\
\%\end{array}$} & \multirow{2}{*}{$\begin{array}{c}\text { Fine sand } \\
\%\end{array}$} & \multirow{2}{*}{ Texture } \\
\hline & & $\mathbf{N}$ & $\mathbf{P}$ & $\mathbf{K}$ & & & & & & & \\
\hline \multirow{2}{*}{ Site 1} & 2018 & 36 & 25 & 78 & 7.5 & 1.23 & Non-saline site & 51.26 & 29.45 & 19.29 & clay loam \\
\hline & 2019 & 34 & 20 & 68 & 7.8 & 1.27 & Non-saline site & 53.62 & 31.24 & 15.14 & clay loam \\
\hline \multirow{2}{*}{ Site 2} & 2018 & 32 & 30 & 68 & 4.8 & 6.27 & Moderately-saline site & 59.65 & 32.56 & 7.79 & clay loam \\
\hline & 2019 & 29 & 35 & 78 & 7.9 & 6.98 & Moderately-saline site & 57.62 & 35.29 & 7.09 & clay loam \\
\hline \multirow{2}{*}{ Site 3} & 2018 & 34 & 40 & 87 & 7.3 & 8.69 & Highly-saline site & 49.67 & 27.52 & 22.81 & clay loam \\
\hline & 2019 & 31 & 35 & 92 & 7.6 & 9.67 & Highly-saline site & 53.49 & 29.67 & 16.84 & clay loam \\
\hline \multicolumn{12}{|c|}{ Post-harvesting } \\
\hline \multirow{2}{*}{ Sites } & \multirow{2}{*}{ Seasons } & \multicolumn{3}{|c|}{ Available } & \multirow{2}{*}{$\mathbf{p H}$} & \multirow{2}{*}{$\mathrm{EC} \mathrm{mmh/cm}$} & \multirow{2}{*}{ Sites category } & \multirow{2}{*}{ Clay \% } & \multirow{2}{*}{$\begin{array}{c}\text { Silt } \\
\%\end{array}$} & \multirow{2}{*}{$\begin{array}{c}\text { Fine sand } \\
\%\end{array}$} & \multirow{2}{*}{ Texture } \\
\hline & & $\mathbf{N}$ & $\mathbf{P}$ & $\mathbf{K}$ & & & & & & & \\
\hline \multirow{2}{*}{ Site 1} & 2018 & 27 & 15 & 57 & 7.4 & 1.19 & Non-saline site & 54.23 & 23.25 & 22.52 & clay loam \\
\hline & 2019 & 25 & 15 & 48 & 7.6 & 1.24 & Non-saline site & 51.42 & 32.22 & 16.36 & clay loam \\
\hline \multirow{2}{*}{ Site 2} & 2018 & 23 & 20 & 44 & 4.6 & 6.22 & Moderately-saline site & 56.43 & 35.51 & 8.06 & clay loam \\
\hline & 2019 & 23 & 30 & 65 & 7.6 & 6.65 & Moderately-saline site & 54.59 & 31.23 & 14.18 & clay loam \\
\hline \multirow{2}{*}{ Site 3} & 2018 & 25 & 30 & 72 & 7.1 & 8.42 & Highly-saline site & 52.46 & 23.46 & 24.08 & clay loam \\
\hline & 2019 & 23 & 20 & 78 & 7.3 & 9.22 & Highly-saline site & 56.32 & 26.26 & 17.42 & clay loam \\
\hline
\end{tabular}

\section{Experimental design}

For each site i.e., non-saline site, moderatelysaline site and highly-saline site, the experiments were performed in a randomized complete block design (RCBD) using split-plot arrangement with three replications. Three levels of potassium fertilizer i.e., 0,25 and $50 \mathrm{~kg} \mathrm{~K}_{2} \mathrm{O}$ fed $^{-1}$ were assigned in the main plots and three sesame varieties i.e., Shandaweel 3, Giza 32 and Sohag 1 were arranged in the sub-plots.
Experimental unit area was $9 \mathrm{~m}^{2}$, which consisting of five ridges, the width of each ridge was $60 \mathrm{~cm}$ and its length was $3 \mathrm{~m}$. Assessed sesame varieties were received from Oil Crops Research Department, Field Crop Research Institute, Agricultural Research Center, Egypt.

\section{Sowing and Crop Care}

Assessed sesame varieties were hand-planted on ridges, $60 \mathrm{~cm}$ and distance between hills was 
according to the recommendations of each variety. The sowing date was performed on $3^{\text {rd }}$ June in both seasons. Seedlings of sesame varieties at 15 days after sowing as recommended were thinned to one or two plants per hill to keep the plant density of each variety per hill. All crop recommendations of other agricultural practices were followed.

\section{Data measured}

\section{A-Yield and its components}

Number of days to $50 \%$ flowering as flowering date was registered for each experimental plots. At harvesting, five competitive plants were randomly taken from the $2^{\text {nd }}$ and $4^{\text {th }}$ ridges, to determine seed yield and its attributes such as plant height in $\mathrm{cm}$, fruiting zone length in $\mathrm{cm}$, number of branches per plant, 1000-seed weight in gram and seed weight per plant in gram. Plants in central ridge from each the experimental unit were taken for determining seed yield per $\mathrm{m}^{2}$ and converted to get to seed yield in $\mathrm{kg}$ feddan.

\section{B-Chemical composition contents of sesame seeds}

Seed oil content was determined according to A.O.A.C. (1975). Nitrogen percentage $(\mathrm{N})$ was determined using micro-kjeldahl method (A.O.A.C. 1995), and it was multiplied by 6.25 to get to protein content (Marschner, 1995). Phosphorus percentage $(\mathrm{P})$ was determined with color metrically following the method introduced by Jackson (1967) and potassium percentage (K) with photo metrically determined by using a flame photometer as described by Jackson (1958).

\section{Statistical analysis}

Mean values of the three varieties affected by the three levels of potassium for all studied traits in the three replications across the three sites and the two seasons were analyzed using randomized complete block design (RCBD) using split-plot arrangement. Field experiments at each sites and seasons were subjected to combined analysis to get bilateral and trilateral interactive effects after confirmed from homogeneity of error variance for these experiments (Gomez and Gomez, 1984).

Means of treatments were compared using the least differences values (L.S.D 5\%) and Duncan's Multiple Range Test at 5\% level of probability according to Gomez and Gomez (1984).

\section{Results and Discussion}

\section{1-Variance analysis for all the studied traits}

All the studied traits of the three sesame varieties evaluated under potassium fertilizer effect across sites and seasons are presented in Table 2. Main effect of year and its dual and triple interactions with other factors were insignificant for most studied traits as shown in the combined analysis, hence it has been ignored. Thus, the discussion focused on the main effects of sites, potassium fertilizer levels, sesame genotypes and their dual and triple interactions. The main effects in combined analysis for sites, potassium fertilizer levels and sesame varieties were highly significant for all the studied traits, indicating that these main effects had considerable effect on all studied traits. As seen in the combined analysis (Table 2), the dual interaction between sites and potassium fertilizer levels was highly significant for all the studied traits. This indicated that the effect of evaluated sites on the studied traits significantly differed from level of potassium fertilizer to another. Similarly, highly significant interaction of evaluated sites with sesame varieties as in combined analysis was observed for all the studied traits. This indicated that effect of sites on the studied traits valuable varied from variety to another. Moreover, as given in the combined analysis, the dual interaction between potassium fertilizer levels and sesame varieties was highly significant for all studied traits. The triple interaction among sites, potassium fertilizer levels and sesame 
varieties was, also as in combined analysis, highly significant with respect to most studied traits. This indicated that effect of sites on the studied traits significantly varied with different levels of potassium fertilizer and sesame varieties.

Table 2. Combined analysis of variance for three sesame varieties as affected by three levels of potassium across three sites and two years regarding all studied traits

\begin{tabular}{|c|c|c|c|c|c|c|c|c|}
\hline \multirow{2}{*}{ S.O.V } & \multicolumn{2}{|l|}{ 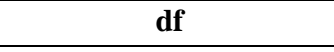 } & $1^{\text {st }}$ season & $2^{\text {nd }}$ season & Combined & $1^{\text {st }}$ season & $2^{\text {nd }}$ season & Combined \\
\hline & Individual & Combined & \multicolumn{3}{|c|}{ Days to $50 \%$ flowering } & \multicolumn{3}{|c|}{ Plant height } \\
\hline Year $(Y)$ & & 1 & & & $107.56^{* *}$ & & & $239.56^{*}$ \\
\hline Site (S) & 2 & 4 & $420.11 * *$ & $347.81 * *$ & $383.96 * *$ & $1570.31 * *$ & $1352.99 * *$ & $1461.65 * *$ \\
\hline Reps within Site & 6 & 12 & 0.74 & 1.05 & 1.55 & 1.64 & 2.26 & 4.49 \\
\hline Potassium (P) & 2 & 2 & $159.37 * *$ & $114.37 * *$ & $270.50 * *$ & $3993.35 * *$ & $3176.06^{* *}$ & $7144.18 * *$ \\
\hline $\mathbf{Y} \times \mathbf{P}$ & & 2 & & & 3.24 & & & 25.23 \\
\hline $\mathbf{S} \times \mathbf{P}$ & 4 & 8 & $12.76^{* *}$ & $16.52 * *$ & $14.64 * *$ & $228.12 * *$ & $253.67 * *$ & $240.89 * *$ \\
\hline Pooled Error a & 12 & 24 & 2.35 & 1.71 & 2.03 & 31.31 & 34.33 & 32.82 \\
\hline Genotypes (G) & 2 & 2 & $124.70 * *$ & $74.93 * *$ & $196.46^{* *}$ & $1377.28 * *$ & $1551.49 * *$ & $2924.59 * *$ \\
\hline $\mathbf{Y} \times \mathbf{G}$ & & 2 & & & 3.17 & & & 4.19 \\
\hline $\mathbf{S} \times \mathbf{G}$ & 4 & 8 & $13.54 * *$ & $13.74 * *$ & $13.64 * *$ & $131.01 * *$ & $131.83 * *$ & $131.42 * *$ \\
\hline $\mathbf{P} \times \mathbf{G}$ & 4 & 4 & $20.52 * *$ & $24.69 * *$ & $43.57 * *$ & $357.06 * *$ & $267.87 * *$ & $616.62 * *$ \\
\hline $\mathbf{S} \times \mathbf{P} \times \mathbf{G}$ & 8 & 4 & $24.49 * *$ & $14.03 * *$ & $19.26^{* *}$ & $116.81^{* *}$ & $106.87 * *$ & $111.84 * *$ \\
\hline $\mathbf{Y} \times \mathbf{P} \times \mathbf{G}$ & & 16 & & & 1.63 & & & 8.31 \\
\hline Pooled Error b & 36 & 72 & 1.31 & 2.12 & 1.71 & 16.71 & 11.91 & 14.31 \\
\hline S.O.V & Individual & Combined & \multicolumn{3}{|c|}{ Fruiting zone length } & \multicolumn{3}{|c|}{ Number of branches per plant } \\
\hline Year $(Y)$ & & 1 & & & $74.56^{* *}$ & & & $18.67 * *$ \\
\hline Site (S) & 2 & 4 & $173.11 * *$ & $684.14 * *$ & $428.63 * *$ & $46.68 * *$ & $49.27 * *$ & $47.98 * *$ \\
\hline Reps within Site & 6 & 12 & 0.80 & 1.47 & 1.92 & 0.26 & 0.55 & 0.46 \\
\hline Potassium (P) & 2 & 2 & $2453.56 * *$ & $2223.49 * *$ & $4674.10 * *$ & $11.20 * *$ & $12.64 * *$ & $23.57 * *$ \\
\hline $\mathbf{Y} \times \mathbf{P}$ & & 2 & & & 2.94 & & & 0.27 \\
\hline $\mathbf{S} \times \mathbf{P}$ & 4 & 8 & $37.37 * *$ & $70.06^{* *}$ & $53.71 * *$ & $1.55^{*}$ & $3.79 * *$ & $2.67 * *$ \\
\hline Pooled Error a & 12 & 24 & 6.16 & 6.95 & 6.55 & 0.43 & 0.50 & 0.47 \\
\hline Genotypes (G) & 2 & 2 & $1414.18 * *$ & $881.53 * *$ & $2264.31 * *$ & $112.75^{* *}$ & $177.42 * *$ & $286.46^{* *}$ \\
\hline $\mathbf{Y} \times \mathbf{G}$ & & 2 & & & 31.40 & & & 3.71 \\
\hline $\mathbf{S} \times \mathbf{G}$ & 4 & 8 & $52.27 * *$ & $111.39 * *$ & $81.83^{* *}$ & $11.99 * *$ & $15.90 * *$ & $13.95 * *$ \\
\hline $\mathbf{P} \times \mathbf{G}$ & 4 & 4 & $183.24 * *$ & $291.93 * *$ & $428.83 * *$ & $6.57 * *$ & $7.60 * *$ & $13.81 * *$ \\
\hline $\mathbf{S} \times \mathbf{P} \times \mathbf{G}$ & 8 & 4 & $66.29 * *$ & $86.67 * *$ & $76.48 * *$ & $2.98 * *$ & $3.03 * *$ & $3.00 * *$ \\
\hline $\mathbf{Y} \times \mathbf{P} \times \mathbf{G}$ & 16 & & & & 46.33 & & & 0.36 \\
\hline Pooled Error b & 36 & 72 & 5.99 & 6.14 & 6.06 & 0.51 & 0.75 & 0.63 \\
\hline S.O.V & Individual & Combined & \multicolumn{3}{|c|}{ 1000-seed weight } & \multicolumn{3}{|c|}{ Seed weight per plant } \\
\hline Year $(Y)$ & & 1 & & & $1.12 * *$ & & & $33.67 * *$ \\
\hline Site (S) & 2 & 4 & $0.50 * *$ & $1.36 * *$ & $0.93 * *$ & $2795.69 * *$ & $2777.88 * *$ & $2786.78 * *$ \\
\hline Reps within Site & 6 & 12 & 0.02 & 0.003 & 0.02 & 1.14 & 1.59 & 1.89 \\
\hline Potassium (P) & 2 & 2 & $4.15^{* *}$ & $3.08 * *$ & $7.19 * *$ & $49.55^{* *}$ & $61.62 * *$ & $110.84 * *$ \\
\hline $\mathbf{Y} \times \mathbf{P}$ & & 2 & & & 0.04 & & & 0.33 \\
\hline $\mathbf{S} \times \mathbf{P}$ & 4 & 8 & $0.05^{*}$ & $0.09 *$ & $0.07 * *$ & $5.19^{*}$ & $10.66^{* *}$ & $7.92 * *$ \\
\hline Pooled Error a & 12 & 24 & 0.01 & 0.02 & 0.01 & 0.97 & 1.01 & 0.99 \\
\hline Genotypes (G) & 2 & 2 & $1.91 * *$ & $0.97 * *$ & $2.80 * *$ & $106.15^{* *}$ & $91.32 * *$ & $196.90 * *$ \\
\hline $\mathbf{Y} \times \mathbf{G}$ & & 2 & & & 0.08 & & & 0.57 \\
\hline $\mathbf{S} \times \mathbf{G}$ & 4 & 8 & $0.08 * *$ & $0.17 * *$ & $0.12 * *$ & $8.04 * *$ & $9.02 * *$ & $8.53 * *$ \\
\hline $\mathbf{P} \times \mathbf{G}$ & 4 & 4 & $0.14 * *$ & $0.07^{*}$ & $0.17 * *$ & $9.72 * *$ & $8.01 * *$ & $15.08 * *$ \\
\hline $\mathbf{S} \times \mathbf{P} \times \mathbf{G}$ & 8 & 4 & $0.05 * *$ & $0.18 * *$ & $0.11 * *$ & 2.11 & $9.96 * *$ & $6.04 * *$ \\
\hline $\mathbf{Y} \times \mathbf{P} \times \mathbf{G}$ & 16 & & & & 0.032 & & & 2.65 \\
\hline Pooled Error b & 36 & 72 & 0.01 & 0.02 & 0.02 & 1.63 & 1.10 & 1.37 \\
\hline
\end{tabular}


Table 2: Continued

\begin{tabular}{|c|c|c|c|c|c|c|c|c|}
\hline \multirow{2}{*}{ S.O.V } & \multicolumn{2}{|c|}{ df } & $1^{\text {st }}$ season & $2^{\text {nd }}$ season & Combined & $1^{\text {st }}$ season & $2^{\text {nd }}$ season & Combined \\
\hline & Individual & Combined & \multicolumn{3}{|c|}{ Seed yield per feddan } & \multicolumn{3}{|c|}{ Seed oil content } \\
\hline Year (Y) & & 1 & & & $9878.09^{* *}$ & & & 1.98 \\
\hline Site (S) & 2 & 4 & $954916.48 * *$ & $1039193.7 * *$ & $997055.12 * *$ & $36.76 * *$ & $52.40 * *$ & $44.58 * *$ \\
\hline Reps within Site & 6 & 12 & 68.19 & 105.01 & 96.41 & 0.48 & 0.69 & 0.81 \\
\hline Potassium $(\mathbf{P})$ & 2 & 2 & $14474.91 * *$ & $20985.17 * *$ & $35155.66 * *$ & $124.19 * *$ & $83.46^{* *}$ & $205.24 * *$ \\
\hline $\mathbf{Y} \times \mathbf{P}$ & & 2 & & & 304.42 & & & 2.41 \\
\hline $\mathbf{S} \times \mathbf{P}$ & 4 & 8 & $1801.30 * *$ & $4471.25 * *$ & $3136.28 * *$ & 2.10 & $8.44 * *$ & $5.27 * *$ \\
\hline Pooled Error a & 12 & 24 & 153.03 & 356.70 & 254.87 & 0.78 & 1.08 & 0.93 \\
\hline Genotypes (G) & 2 & 2 & $42071.07 * *$ & $31750.58 * *$ & $73458.11 * *$ & $87.53 * *$ & $57.39 * *$ & $141.62 * *$ \\
\hline $\mathbf{Y} \times \mathbf{G}$ & & 2 & & & 363.54 & & & 3.30 \\
\hline $\mathbf{S} \times \mathbf{G}$ & 4 & 8 & $2998.61 * *$ & $3925.82 * *$ & $3462.21 * *$ & $3.03 * *$ & $3.78 *$ & $3.40 * *$ \\
\hline $\mathbf{P} \times \mathbf{G}$ & 4 & 4 & $3967.61 * *$ & $2434.85 * *$ & $4965.23 * *$ & $4.06 * *$ & $8.17 * *$ & $11.49 * *$ \\
\hline $\mathbf{S} \times \mathbf{P} \times \mathbf{G}$ & 8 & 4 & $467.21 *$ & $3803.65 * *$ & $2135.43 * *$ & 1.49 & $4.31 * *$ & $2.90 * *$ \\
\hline $\mathbf{Y} \times \mathbf{P} \times \mathbf{G}$ & & 16 & & & 1437.23 & & & 0.74 \\
\hline Pooled Error b & 36 & 72 & 181.51 & 145.58 & 163.54 & 0.71 & 1.08 & 0.89 \\
\hline S.O.V & Individual & Combined & \multicolumn{3}{|c|}{ Seed protein content } & \multicolumn{3}{|c|}{ Seed nitrogen content } \\
\hline Year $(Y)$ & & 1 & & & 0.18 & & & 0.005 \\
\hline Site (S) & 2 & 4 & $49.73 * *$ & $50.30 * *$ & $50.02 * *$ & $1.27 * *$ & $1.29 * *$ & $1.28 * *$ \\
\hline Reps within Site & 6 & 12 & 0.08 & 0.09 & 0.11 & 0.002 & 0.002 & 0.003 \\
\hline Potassium (P) & 2 & 2 & $10.48 * *$ & $9.86^{* *}$ & $20.34 * *$ & $0.27 * *$ & $0.25 * *$ & $0.52 * *$ \\
\hline $\mathbf{Y} \times \mathbf{P}$ & & 2 & & & 0.01 & & & 0.0002 \\
\hline $\mathbf{S} \times \mathbf{P}$ & 4 & 8 & $1.82 * *$ & $1.39 * *$ & $1.60 * *$ & $0.05 * *$ & $0.04 * *$ & $0.04 * *$ \\
\hline Pooled Error a & 12 & 24 & 0.18 & 0.09 & 0.14 & 0.005 & 0.002 & 0.004 \\
\hline Genotypes (G) & 2 & 2 & $5.48 * *$ & $4.53 * *$ & $9.99 * *$ & $0.14 * *$ & $0.12 * *$ & $0.26 * *$ \\
\hline $\mathbf{Y} \times \mathbf{G}$ & & 2 & & & 0.02 & & & 0.0006 \\
\hline $\mathbf{S} \times \mathbf{G}$ & 4 & 8 & $0.25 *$ & $0.28 *$ & $0.26 * *$ & $0.01 *$ & $0.01 *$ & $0.01 * *$ \\
\hline $\mathbf{P} \times \mathbf{G}$ & 4 & 4 & $0.25 *$ & 0.18 & $0.42 * *$ & $0.01 *$ & 0.005 & $0.01 * *$ \\
\hline $\mathbf{S} \times \mathbf{P} \times \mathbf{G}$ & 8 & 4 & $0.46^{* *}$ & $0.49 * *$ & $0.48 * *$ & $0.01 * *$ & $0.01 * *$ & 0.0005 \\
\hline $\mathbf{Y} \times \mathbf{P} \times \mathbf{G}$ & 16 & & & & 0.02 & & & $0.01 * *$ \\
\hline Pooled Error b & 36 & 72 & 0.09 & 0.08 & 0.09 & 0.002 & 0.002 & 0.002 \\
\hline S.O.V & Individual & Combined & \multicolumn{3}{|c|}{ Seed phosphorous content } & \multicolumn{3}{|c|}{ Seed potassium content } \\
\hline Year $(Y)$ & & 1 & & & 0.001 & & & 0.001 \\
\hline Site (S) & 2 & 4 & $0.29 * *$ & $0.26^{* *}$ & $0.27 * *$ & $1.23 * *$ & $1.58 * *$ & $1.41 * *$ \\
\hline Reps within Site & 6 & 12 & 0.0003 & 0.0002 & 0.0004 & 0.002 & 0.003 & 0.003 \\
\hline Potassium (P) & 2 & 2 & $0.06 * *$ & $0.05 * *$ & $0.11 * *$ & $0.42 * *$ & $0.45^{* *}$ & $0.87 * *$ \\
\hline $\mathbf{Y} \times \mathbf{P}$ & & 2 & & & 0.0004 & & & 0.003 \\
\hline $\mathbf{S} \times \mathbf{P}$ & 4 & 8 & $0.01 * *$ & $0.01 * *$ & $0.01 * *$ & $0.05 * *$ & $0.05 * *$ & $0.05 * *$ \\
\hline Pooled Error a & 12 & 24 & 0.0005 & 0.0002 & 0.0003 & 0.004 & 0.003 & 0.004 \\
\hline Genotypes (G) & 2 & 2 & $0.03 * *$ & $0.04 * *$ & $0.07 * *$ & $0.10 * *$ & $0.16^{* *}$ & $0.25 * *$ \\
\hline $\mathbf{Y} \times \mathbf{G}$ & & 2 & & & 0.001 & & & 0.01 \\
\hline $\mathbf{S} \times \mathbf{G}$ & 4 & 8 & $0.001 *$ & $0.002 * *$ & $0.001 * *$ & $0.04 * *$ & $0.04 * *$ & $0.04 * *$ \\
\hline $\mathbf{P} \times \mathbf{G}$ & 4 & 4 & $0.002 * *$ & $0.01 * *$ & $0.01 * *$ & $0.01 * *$ & $0.02 * *$ & $0.03 * *$ \\
\hline $\mathbf{S} \times \mathbf{P} \times \mathbf{G}$ & 8 & 4 & $0.003 * *$ & $0.005 * *$ & $0.004 * *$ & $0.04 * *$ & $0.04 * *$ & $0.04 * *$ \\
\hline $\mathbf{Y} \times \mathbf{P} \times \mathbf{G}$ & 16 & & & & 0.002 & & & 0.002 \\
\hline Pooled Error b & 36 & 72 & 0.0004 & 0.0003 & 0.0004 & 0.002 & 0.003 & 0.003 \\
\hline
\end{tabular}

* and **, indicate significant at 0.05 and 0.01 levels of probability, respectively.

2-Main effects

2-1-Sites effects

2-1-A- Yield and its components
Evaluated sites had significant effect on all the studied traits (Table 3). This might be attributed to these sites had different degrees of soil salinity as presented in Table 1. As revealed in the mean 
values of pooled analysis, apparently, increasing the degree of salinity soil with sowing at nonsaline to moderately-saline site then up to strongly-saline site was associated with decreased in all studied traits. These reduction percentages were $3.69 \%$ and $11.57 \%$ for days to $50 \%$ flowering, $1.65 \%$ and $7.83 \%$ for plant height, $4.59 \%$ and $4.18 \%$ for fruiting zone length, $32.21 \%$ and $53.15 \%$ for number of branches per plant, $0.56 \%$ and $8.14 \%$ for 1000 seed weight, $31.35 \%$ and $62.16 \%$ for seed weight per plant, and $31.34 \%$ and $61.86 \%$ for seed yield per feddan under moderately-saline site and strongly-saline site with compared to non-saline site, respectively. Similar results were reported by Nóbrega et al. (2018) and Vadaliya et al. (2019). These results could be traced back to sesame plants, under moderate to high salinity soil conditions, tried to escape this salt stress through shortening their life cycle in the form of early flowering. The graded saline stress from low to medium up to high at evaluated sites had the greatest negative impact on plant height, fruiting zone length and number of branches per plant as vegetative growth, hence these reduction reflected on 1000-seed weight, seed weight per plant and seed yield per feddan through inhibiting the growth of root and its ability to water uptake and needed mineral nutrition from soil also declined rate of leaf creation, hence low number of leaves leading to shrank photosynthesis (Puvanitha and Mahendran, 2017) and other physiological processes in plant with discouraging transfer of photosynthesis products from leaves to the rest parts of plant, especially the economic ones such as seeds as sink (Desingh and Kanagaraj, 2020).

Table 3. The main effect of salt-affected sites on seed yield and its components as combined analysis across the studied sesame varieties, potassium levels and the two seasons

\begin{tabular}{|c|c|c|c|c|c|c|c|}
\hline Traits & $\begin{array}{c}\text { Days to } \\
50 \% \\
\text { flowering } \\
\left(\mathbf{N}^{0}\right)\end{array}$ & $\begin{array}{c}\text { Plant } \\
\text { height } \\
(\mathbf{c m})\end{array}$ & $\begin{array}{l}\text { Fruiting } \\
\text { zone } \\
\text { length } \\
\text { (cm) }\end{array}$ & $\begin{array}{c}\text { Number of } \\
\text { branches } \\
\text { per plant } \\
\left(\mathbf{N}^{0}\right)\end{array}$ & $\begin{array}{l}\text { 1000-seed } \\
\text { weight }(g)\end{array}$ & $\begin{array}{c}\text { Seed } \\
\text { weight per } \\
\text { plant }(g)\end{array}$ & $\begin{array}{l}\text { Seed yield } \\
\text { per feddan } \\
\quad(\mathrm{kg})\end{array}$ \\
\hline Non-saline site & $63.69^{\mathrm{a}}$ & $178.19^{\mathrm{a}}$ & $89.99^{\mathrm{a}}$ & $4.94^{\mathrm{a}}$ & $3.97^{\mathrm{a}}$ & $32.69^{\mathrm{a}}$ & $621.13^{\mathrm{a}}$ \\
\hline Moderately-saline site & $61.33^{\mathrm{b}}$ & $175.24^{\mathrm{b}}$ & $85.86^{\mathrm{b}}$ & $3.35^{\mathrm{b}}$ & $3.94^{\mathrm{b}}$ & $22.44^{\mathrm{b}}$ & $426.47^{b}$ \\
\hline Highly-saline site & $56.31^{\mathrm{c}}$ & $164.24^{\mathrm{c}}$ & $82.63^{\mathrm{c}}$ & $2.31^{\mathrm{c}}$ & $3.64^{\mathrm{c}}$ & $12.37^{\mathrm{c}}$ & $236.90^{c}$ \\
\hline LSD 0.05 & 0.57 & 3.22 & 1.44 & 0.38 & 0.07 & 0.56 & 8.97 \\
\hline
\end{tabular}

Means followed by the same letter are not significantly different from one another based on Duncan's multiple test at $5 \%$.

\section{2-1-B- Chemical composition contents of sesame seeds}

Significant effect of evaluated sites on seed oil, protein, nitrogen, phosphorus and potassium contents was observed (Table 4). This might be attributed to these sites had different degrees of soil salinity as presented in Table 1. As presented in the pooled analysis, clearly, increasing the degree of salinity soil with sowing at non-saline to moderately-saline site then up to strongly-saline site associated with decreased in all these traits. These reduction percentages were $3.15 \%$ and $5.25 \%$ for seed oil content, $5.94 \%$ and $15.93 \%$ for seed protein content, $5.94 \%$ and
$15.93 \%$ for seed nitrogen content, $21.88 \%$ and $56.08 \%$ for seed phosphorus content and $8.82 \%$ and $14.37 \%$ for potassium content when sowing at moderately-saline site and strongly-saline site with compared to non-saline site, respectively. Similar findings were reported by Nóbrega et al. (2018) and Vadaliya et al. (2019). High concentrations of salt force both osmotic and ionic stresses on the plants and have an effect on plant growth by changing their morphological, anatomical and physiological traits. Many researchers registered increasing salinity induced decrease in the progress of the xylem (Puvanitha and Mahendran, 2017). 
Table 4. The main effect of salt-affected sites on chemical composition contents of sesame seeds as combined analysis across the studied sesame varieties, potassium levels and the two seasons

\begin{tabular}{|c|c|c|c|c|c|}
\hline Site ${ }^{\text {Traits }}$ & $\begin{array}{c}\text { Seed oil content } \\
(\%)\end{array}$ & $\begin{array}{l}\text { Seed protein } \\
\text { content }(\%)\end{array}$ & $\begin{array}{l}\text { Seed nitrogen } \\
\text { content }(\%)\end{array}$ & $\begin{array}{l}\text { Seed phosphorus } \\
\text { content }(\%)\end{array}$ & $\begin{array}{l}\text { Seed potassium } \\
\text { content }(\%)\end{array}$ \\
\hline Non-saline site & $48.35^{\mathrm{a}}$ & $16.91^{\mathrm{a}}$ & $2.70^{\mathrm{a}}$ & $0.36^{\mathrm{a}}$ & $3.11^{\mathrm{a}}$ \\
\hline Moderately-saline site & $46.82^{\mathrm{b}}$ & $15.90^{\mathrm{b}}$ & $2.54^{\mathrm{b}}$ & $0.28^{\mathrm{b}}$ & $2.96^{\mathrm{b}}$ \\
\hline Highly saline site & $45.81^{\mathrm{c}}$ & $14.21^{\mathrm{c}}$ & $2.27^{\mathrm{c}}$ & $0.16^{\mathrm{c}}$ & $2.67^{\mathrm{c}}$ \\
\hline LSD 0.05 & 0.54 & 0.21 & 0.03 & 0.01 & 0.03 \\
\hline
\end{tabular}

Means followed by the same letter are not significantly different from one another based on Duncan's multiple test at $5 \%$.

\section{2-2-Potassium fertilizer effect}

\section{2-2-A- Yield and its components}

Application of potassium fertilizer with graded levels from zero to 25 up to $50 \mathrm{~kg} \mathrm{~K}_{2} \mathrm{O}$ fed $^{-1}$ accompanied by a gradual increasing in the all studied traits (Table 5). In this respect, increasing application of potassium fertilizer at levels of 25 and $50 \mathrm{~kg} \mathrm{~K}_{2} \mathrm{O}$ fed ${ }^{-1}$ comparing nonapplication showed an increase of studied parameters as follow; $1.13 \%$ and $7.08 \%$ for days to $50 \%$ flowering, $10.99 \%$ and $13.58 \%$ for plant height, $13.01 \%$ and $24.26 \%$ for fruiting zone length, $4.19 \%$ and $38.92 \%$ for number of branches per plant, $7.76 \%$ and $20.54 \%$ for 1000 -seed weight, $8.02 \%$ and $13.58 \%$ for seed weight per plant, and $7.26 \%$ and $12.67 \%$ for seed yield per feddan, respectively. Similar results were found by Hafiz and El-Bramawy (2012), Abdel-Rahman (2014), Jat et al. (2017) and Ahmad et al. (2018). The positive effect of potassium fertilizer on all studied traits might be due to the vital role of potassium in carbohydrates synthesis, photosynthesis and cell elongation as well as its role in the assimilates translocation from source to sink. Through its function as an activator of many enzymatic responses and in electrochemical activities, potassium shows a basic role in assimilation, phloem loading and long-distance assimilates transport, in nitrogen $(\mathrm{N})$ metabolism and in storage activities. Thus, potassium is essential for yield and quality in plant (Rasul, 2010).

Table 5. The main effect of potassium fertilizer levels on seed yield and its components, as combined analysis across the studied sesame varieties, sites and the two seasons

\begin{tabular}{|c|c|c|c|c|c|c|c|}
\hline Potassium fertilizer leveh & $\begin{array}{c}\text { Days to } \\
50 \% \\
\text { flowering } \\
\left(\mathbf{N}^{0}\right)\end{array}$ & $\begin{array}{c}\text { Plant } \\
\text { height } \\
(\mathrm{cm})\end{array}$ & $\begin{array}{l}\text { Fruiting } \\
\text { zone } \\
\text { length } \\
(\mathrm{cm})\end{array}$ & $\begin{array}{c}\text { Number } \\
\text { of } \\
\text { branches } \\
\text { per plant } \\
\left(\mathbf{N}^{0}\right)\end{array}$ & $\begin{array}{l}\text { 1000-seed } \\
\text { weight (g) }\end{array}$ & $\begin{array}{c}\text { Seed } \\
\text { weight } \\
\text { per plant } \\
\text { (g) }\end{array}$ & $\begin{array}{c}\text { Seed yield } \\
\text { per } \\
\text { feddan } \\
(\mathbf{k g})\end{array}$ \\
\hline $\mathrm{kg} \mathrm{K}_{2} \mathrm{O}$ fed $^{-1}$ & $58.83^{\mathrm{c}}$ & $159.49^{\mathrm{c}}$ & $76.64^{\mathrm{c}}$ & $3.09^{\mathrm{c}}$ & $3.52^{\mathrm{c}}$ & $20.99^{\mathrm{c}}$ & $401.52^{c}$ \\
\hline $\operatorname{kg~K}_{2} O$ fed $^{-1}$ & $59.50^{\mathrm{b}}$ & $177.02^{\mathrm{b}}$ & $86.61^{\mathrm{b}}$ & $3.22^{\mathrm{b}}$ & $3.79^{\mathrm{b}}$ & $22.67^{\mathrm{b}}$ & $430.60^{\mathrm{b}}$ \\
\hline $\mathrm{kg} \mathrm{K}_{2} \mathrm{O}$ fed $^{-1}$ & $63.00^{\mathrm{a}}$ & $181.16^{\mathrm{a}}$ & $95.23^{\mathrm{a}}$ & $4.30^{\mathrm{a}}$ & $4.24^{\mathrm{a}}$ & $23.84^{\mathrm{a}}$ & $452.38^{\mathrm{a}}$ \\
\hline LSD 0.05 & 0.57 & 3.22 & 1.44 & 0.38 & 0.07 & 0.56 & 8.97 \\
\hline
\end{tabular}

Means followed by the same letter are not significantly different from one another based on Duncan's multiple test at $5 \%$.

2-2-B- Chemical composition contents of sesame seeds

Application gradient of potassium fertilizer from zero to 25 up to $50 \mathrm{~kg} \mathrm{~K}_{2} \mathrm{O}$ fed $^{-1}$ per feddan associated with a gradual increasing in seed oil, protein, nitrogen, phosphorus and potassium contents (Table 6). In this respect, both potassium level of 25 and $50 \mathrm{~kg} \mathrm{~K}_{2} \mathrm{O}$ fed $^{-1}$ exceeded the control treatment by $3.23 \%$ and $8.54 \%$ for seed oil content, $5.78 \%$ and $7.92 \%$ for seed protein content, $5.78 \%$ and $7.92 \%$ for seed nitrogen content, $32.15 \%$ and $39.01 \%$ for seed phosphorous content and $7.89 \%$ and 8.03 
$\%$ for potassium content, respectively (Table 6). Similar results were found by Hafiz and ElBramawy (2012), Abdel-Rahman (2014), Jat et al. (2017) and Ahmad et al. (2018). This might be attributed to potassium fertilizer showed a vital role in plant adjusting development including osmoregulation, plant-water relation and intercalation/anion balance. It also has abundant effect on enzyme activation shared in the formation of organic substances, protein and starch synthesis, respiratory and photosynthetic metabolism (Marschner, 2010) and sucking up of water and nutrients from the soil (Elmasry, 2009).

Table 6. The main effect of potassium fertilizer levels on chemical composition contents of sesame seeds, as combined analysis across the studied sesame varieties, sites and the two seasons

\begin{tabular}{|c|c|c|c|c|c|c|}
\hline \multicolumn{2}{|c|}{ Potassium fertilizer levels } & $\begin{array}{c}\text { Seed oil content } \\
(\%)\end{array}$ & $\begin{array}{l}\text { Seed protein } \\
\text { content }(\%)\end{array}$ & $\begin{array}{l}\text { Seed nitrogen } \\
\text { content }(\%)\end{array}$ & $\begin{array}{c}\text { Seed } \\
\text { phosphorus } \\
\text { content }(\%)\end{array}$ & $\begin{array}{l}\text { Seed potassium } \\
\text { content }(\%)\end{array}$ \\
\hline $\mathbf{0}$ & $\mathrm{kg} \mathrm{K}_{2} \mathrm{O}$ fed $^{-1}$ & $45.22^{\mathrm{c}}$ & $14.99^{\mathrm{c}}$ & $2.40^{\mathrm{c}}$ & $0.21^{\mathrm{c}}$ & $2.77^{b}$ \\
\hline 25 & $\mathrm{~kg} \mathrm{~K}_{2} \mathrm{O} \mathrm{fed}^{-1}$ & $46.68^{b}$ & $15.85^{\mathrm{b}}$ & $2.54^{\mathrm{b}}$ & $0.28^{b}$ & $2.99^{\mathrm{a}}$ \\
\hline 50 & $\mathrm{~kg} \mathrm{~K}_{2} \mathrm{O} \mathrm{fed}^{-1}$ & $49.08^{\mathrm{a}}$ & $16.17^{\mathrm{a}}$ & $2.59^{\mathrm{a}}$ & $0.30^{\mathrm{a}}$ & $2.99^{\mathrm{a}}$ \\
\hline LSD 0.05 & & 0.54 & 0.21 & 0.03 & 0.01 & 0.03 \\
\hline
\end{tabular}

Means followed by the same letter are not significantly different from one another based on Duncan's multiple test at $5 \%$.

\section{2-3--Sesame varieties effects}

\section{2-3-A- Yield and its components}

Sesame varieties significantly differed in their performance regarding studied traits (Table 7). Sohag1 variety had shorter period to $50 \%$ flowering (58.28 day) comparing other varieties. The superiority was in favor of Shandaweel 3 followed by Giza 32 over Sohag 1 for plant height by $8.62 \%$ and $6.43 \%$, fruiting zone length by $16.26 \%$ and $8.30 \%, 1000$-seed weight by $12.14 \%$ and $3.72 \%$ and consequently seed weight per plant by $18.50 \%$ and $8.64 \%$, and seed yield per feddan by $18.87 \%$ and $9.72 \%$ with compared to Sohag1, in respective order. Similar findings were reported by Gaballah et al. (2007), Suassuna et al. (2017) and Vadaliya et al. (2019). Shandaweel 3 possessed the fewest number of branches per plant with compared to the other varieties. The superiority of Shandaweel 3 might be attributed to the efficiency of its roots in absorbing water from the soil and its leaves in the photosynthesis process and its ability to transfer nutrients from the leaves as a source to the remaining parts of the plant, especially economic ones such as seeds as a sink, which has been reflected on yield components and consequently seed weight per plant and seed yield per feddan.

Table 7. Agronomic performance of tested sesame varieties, as combined analysis across potassium fertilizer levels, sites and the two seasons

\begin{tabular}{|c|c|c|c|c|c|c|c|}
\hline Sesame varieties & $\begin{array}{c}\text { Days to } \\
50 \% \\
\text { flowering } \\
\left(\mathbf{N}^{0}\right)\end{array}$ & $\begin{array}{c}\text { Plant } \\
\text { height } \\
(\mathbf{c m})\end{array}$ & $\begin{array}{l}\text { Fruiting } \\
\text { zone } \\
\text { length }(\mathrm{m})\end{array}$ & $\begin{array}{c}\text { Number of } \\
\text { branches } \\
\text { per plant } \\
\left(\mathbf{N}^{0}\right)\end{array}$ & $\begin{array}{l}\text { 1000-seed } \\
\text { weight (g) }\end{array}$ & $\begin{array}{c}\text { Seed } \\
\text { weight per } \\
\text { plant (g) }\end{array}$ & $\begin{array}{l}\text { Seed yield } \\
\text { per feddan } \\
(\mathrm{kg})\end{array}$ \\
\hline Shandaweel 3 & $61.87^{\mathrm{a}}$ & $178.47^{\mathrm{a}}$ & $92.59^{\mathrm{a}}$ & $0.89^{\mathrm{c}}$ & $4.10^{\mathrm{a}}$ & $24.45^{\mathrm{a}}$ & $464.67^{\mathrm{a}}$ \\
\hline Giza 32 & $61.19^{\mathrm{b}}$ & $174.88^{\mathrm{b}}$ & $86.25^{\mathrm{b}}$ & $5.07^{\mathrm{a}}$ & $3.79^{\mathrm{b}}$ & $22.41^{\mathrm{b}}$ & $428.91^{\mathrm{b}}$ \\
\hline Sohag 1 & $58.28^{\mathrm{c}}$ & $164.31^{\mathrm{c}}$ & $79.64^{\mathrm{c}}$ & $4.65^{\mathrm{b}}$ & $3.66^{\mathrm{c}}$ & $20.63^{c}$ & $390.91^{\mathrm{c}}$ \\
\hline LSD 0.05 & 0.50 & 2.05 & 1.34 & 0.43 & 0.07 & 0.63 & 6.94 \\
\hline
\end{tabular}




\section{2-3-B- Chemical composition contents of sesame seeds}

Performance of sesame varieties significantly differed in seed oil, protein and nitrogen $(\mathrm{N})$, phosphorus (P) and potassium (K) contents (Table 8). Shandaweel 3 and Giza 32 variety had the highest proportion of seed phosphorus content by $29.68 \%$ and $9.37 \%$, respectively with compared to Sohag 1 . The superiority was in favor of Shandaweel 3 followed by Giza 32 for seed oil content by $7.06 \%$ and $2.76 \%$, seed protein content by $5.47 \%$ and $1.61 \%$, seed nitrogen content by $5.41 \%$ and $1.61 \%$ and seed potassium content by $4.43 \%$ and $0.67 \%$, respectively over Sohag 1 . Similar findings were reported by Gaballah et al. (2007), Suassuna et al. (2017) and Vadaliya et al. (2019). The superiority of Shandaweel 3 could be attributed to the efficiency of its roots in absorbing water from the soil and its leaves in the photosynthesis process and its ability to transfer nutrients from the leaves as a source to the remaining parts of the plant, especially economic ones such as seeds as a sink, which has been reflected on seed oil, protein and NPK contents.

Table 8. The main effect of sesame varieties on chemical composition contents of sesame seeds, as combined analysis across potassium fertilizer levels, sites and the two seasons

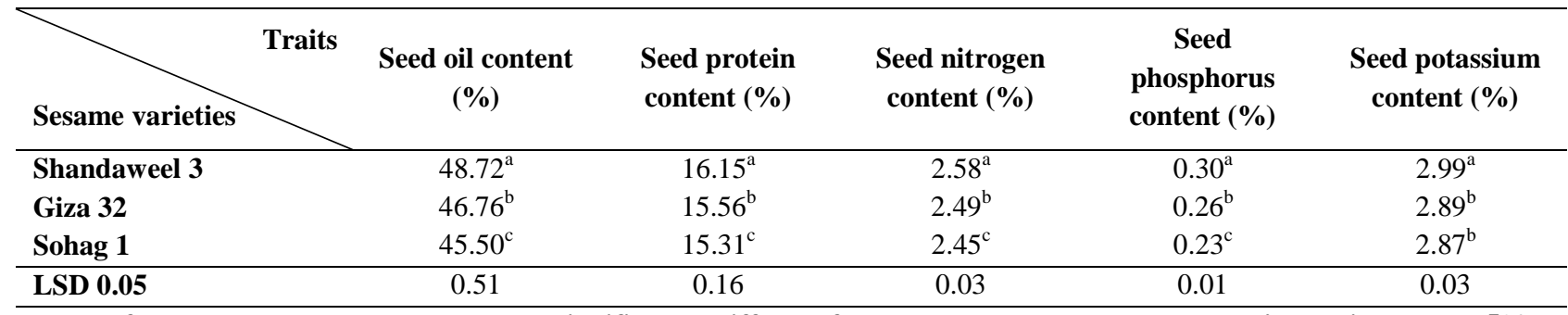

Means followed by the same letter are not significantly different from one another based on Duncan's multiple test at $5 \%$.

\section{3- Dual and triple interactions effects on all studied traits}

The dual interactive of evaluated sites with potassium fertilizer was significant for all studied traits (Table 2). The highest values of studied traits were observed under non-saline site with potassium application at the rate of $50 \mathrm{~kg}$ $\mathrm{K}_{2} \mathrm{O}$ fed $^{-1}$ (Fig.1a-11). Moreover, the interaction effect of increasing the application of potassium under all evaluated sites was effective in improving performance of studied traits. This indicated the gradual increase of potassium fertilizer improved plants capability to salinity tolerance under studied conditions.

The dual interaction of evaluated sites with tested sesame varieties had significant effect on performance of studied traits (Table 2). The highest values of most studied traits were detected when Shandaweel 3 was sown under non-saline site (Fig.1a-11). Moreover, sowing Shandaweel 3 under the moderately and strongly-saline site was superior to other varieties (Fig.1a-11). This might be attributed to its ability to adapt to adverse conditions, such as salt stress. Many studies have revealed that the growth of shoot system is influenced, either negatively or positively by modifications in salinity absorption, type of salt present or type of plant species, and the decrease in plant growth at the highest salinity degrees might be attributed to the inhibition in hydrolysis of reserved foods and their translocation to the growing shoots (Puvanitha and Mahendran, 2017).

The positive and significant interactive of potassium fertilizer with sesame varieties was observed in their all studied traits (Table 2). The highest values of most studied traits were observed when sowing Shandaweel 3 followed by Giza 32 and Sohag 1 with increasing level of 
potassium application up to $50 \mathrm{~K}_{2} \mathrm{O} \mathrm{kg} \mathrm{fed}^{-1}$ (Fig.1a-11).

Moreover, the triple interaction of sites, potassium fertilizer with sesame genotypes had significant effect on all studied traits (Table 2). This gives the opportunity to determine the best triple interactions for all studied traits. From that

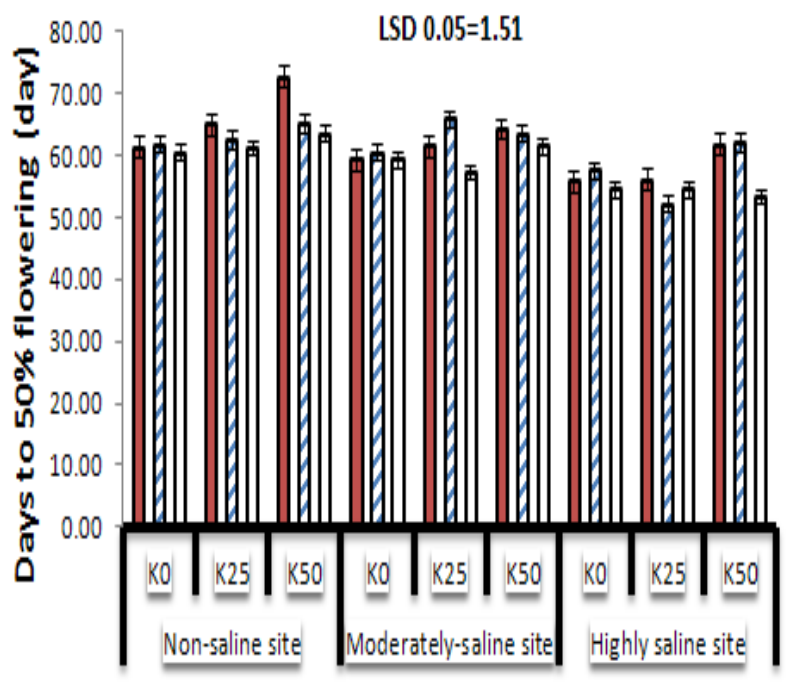

QShandaweel-3 QGiza 32 QSohag 1

a-Days to $50 \%$ flowering, day

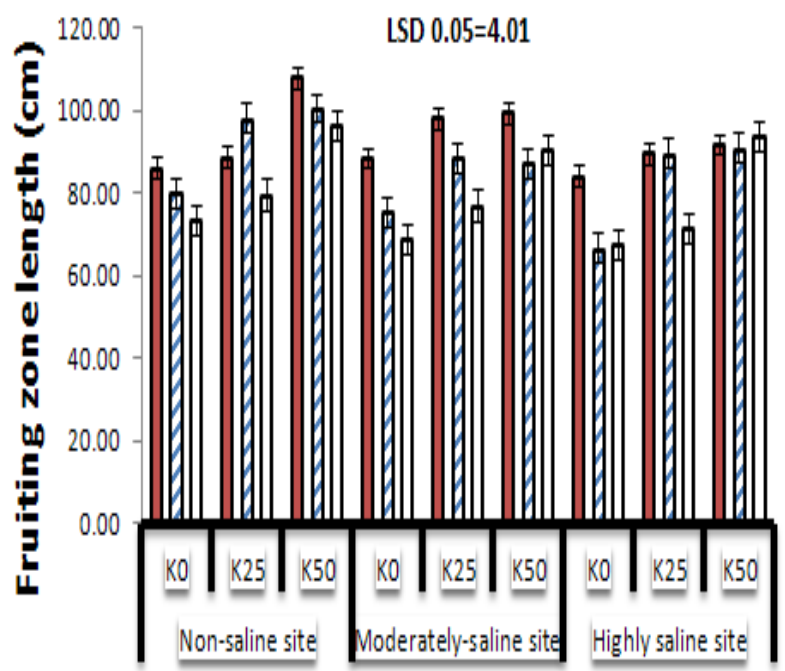

पShandaweel-3 पGiza 32 QSohag 1

c-Fruiting zone length, $\mathrm{cm}$ standpoint, the best triple interaction was obtained by sowing Shandaweel 3 at non-saline site with application potassium fertilizer at level of $50 \quad \mathrm{~K}_{2} \mathrm{O} \mathrm{kg} \mathrm{fed}{ }^{-1}$ (Fig.1a-11). Moreover, Shandaweel 3 was superior to other varieties, even when it was sown at other salt-affected sites with application of potassium at the highest level $50 \mathrm{~K}_{2} \mathrm{O} \mathrm{kg} \mathrm{fed}{ }^{-1}$ (Fig.1a-11).

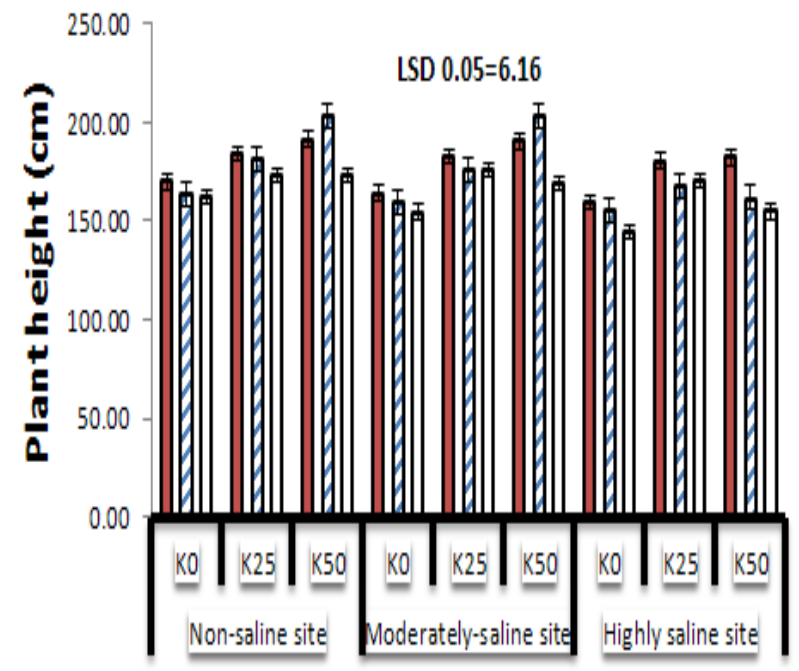

QShandaweel-3 ¿Giza 32 QSohag 1

b-Plant height, cm

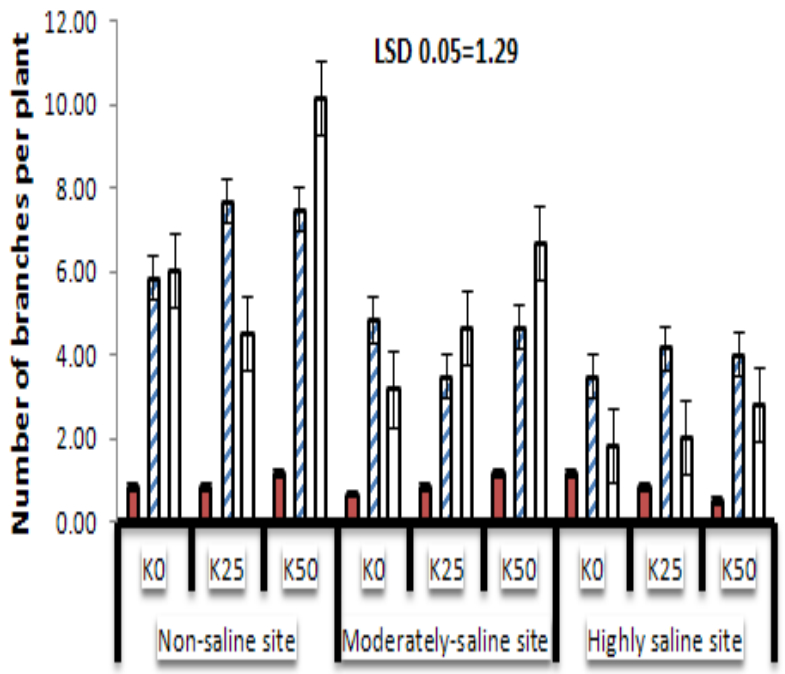

QShandaweel-3 DGiza 32 QSohag 1

d-Number of branches per plant 


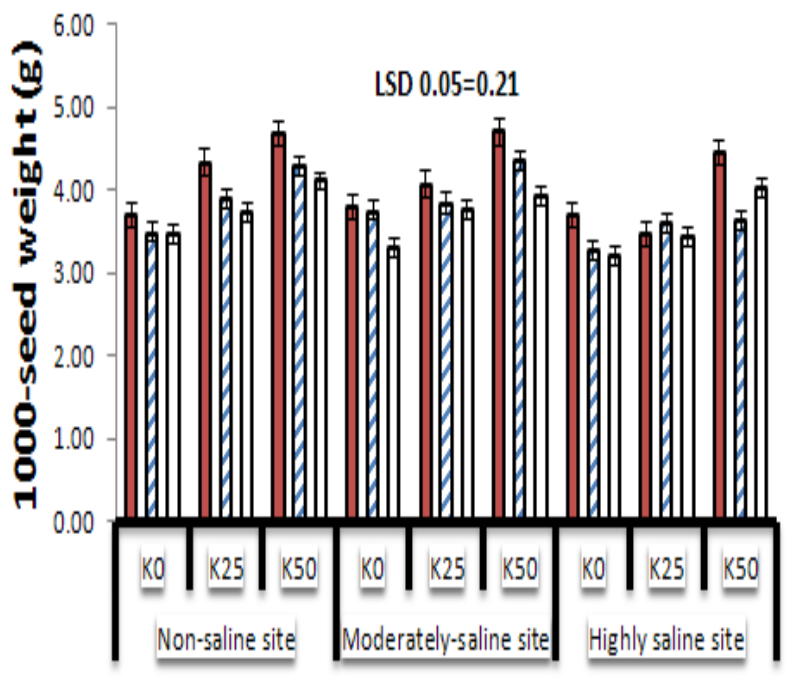

QShandaweel-3 $\quad$ Giza 32 QSohag 1

e-1000-seed weight, $g$

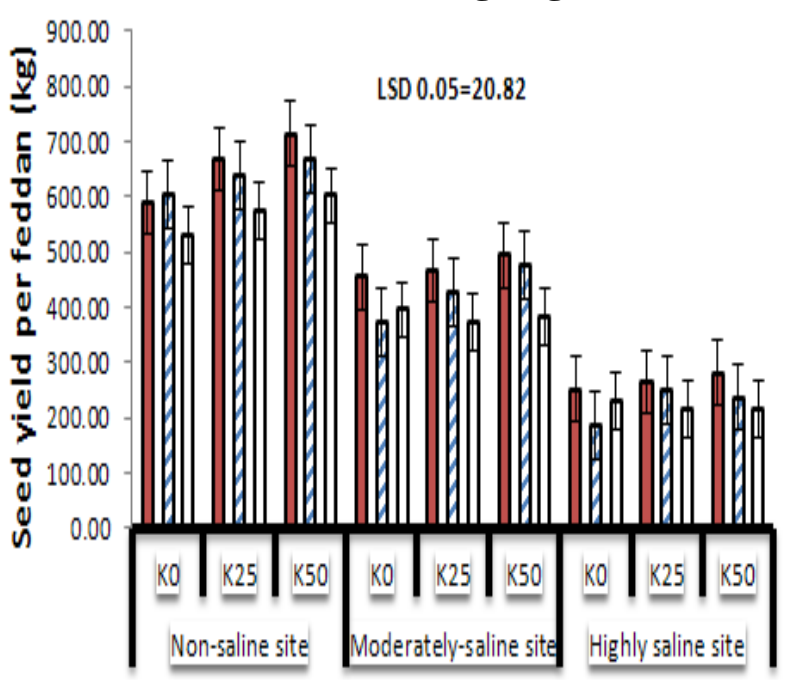

QShandaweel-3 QGiza 32 QSohag 1

g-Seed yield per feddan, $\mathrm{kg}$

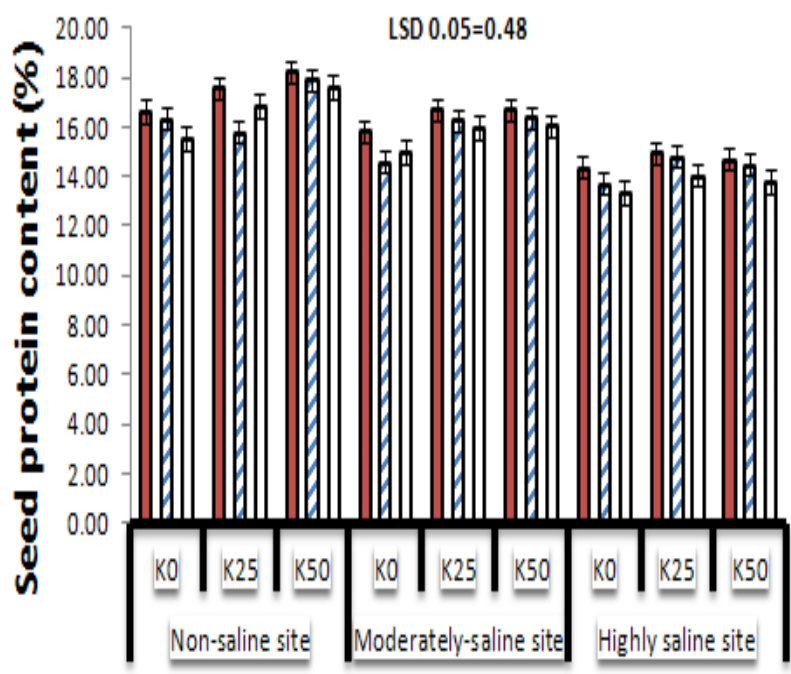

QShandaweel-3 QGiza 32 QSohag 1

i-Seed protein content, \%

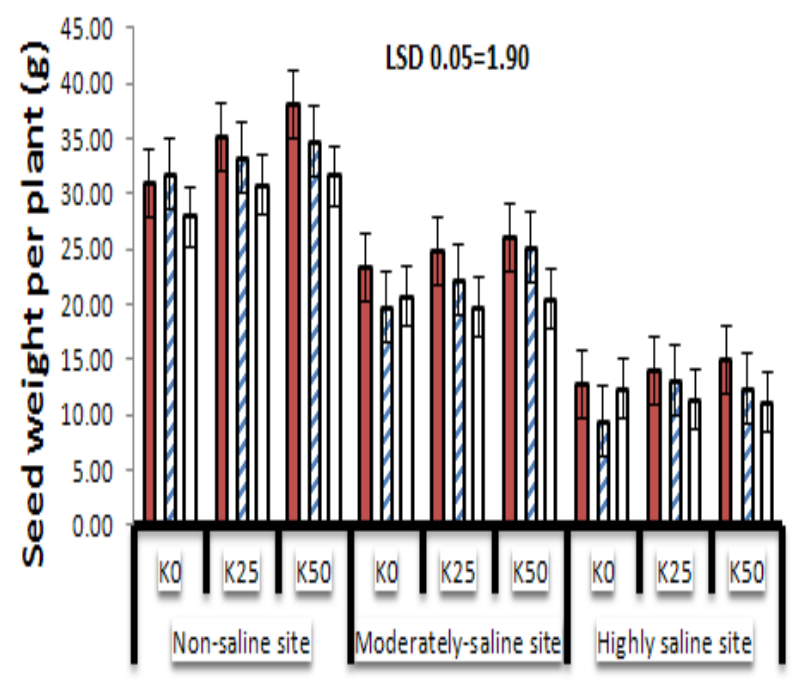

DShandaweel-3 DGiza 32 QSohag 1

f-Seed weight per plant $g$

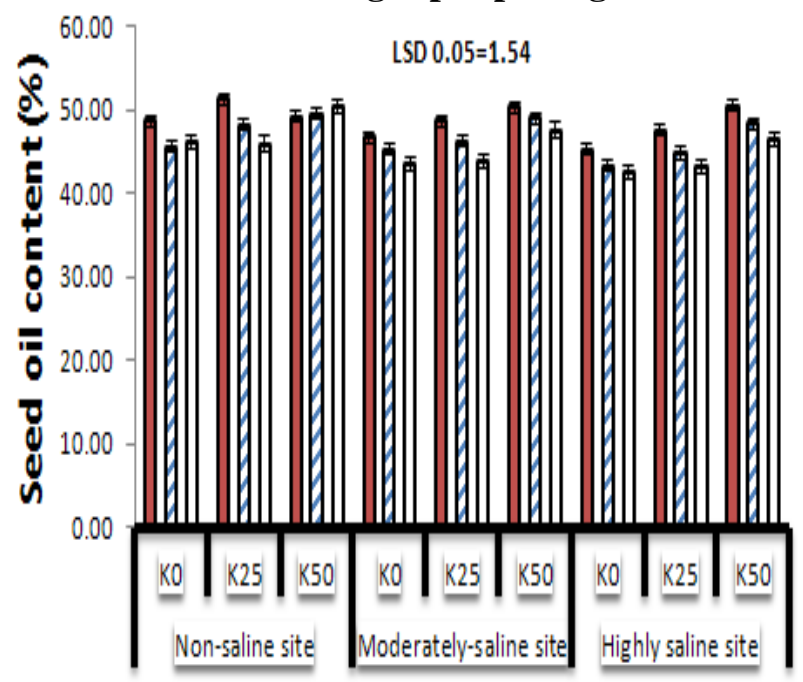

QShandaweel-3 QGiza 32 QSohag 1

h-Seed oil content, \%

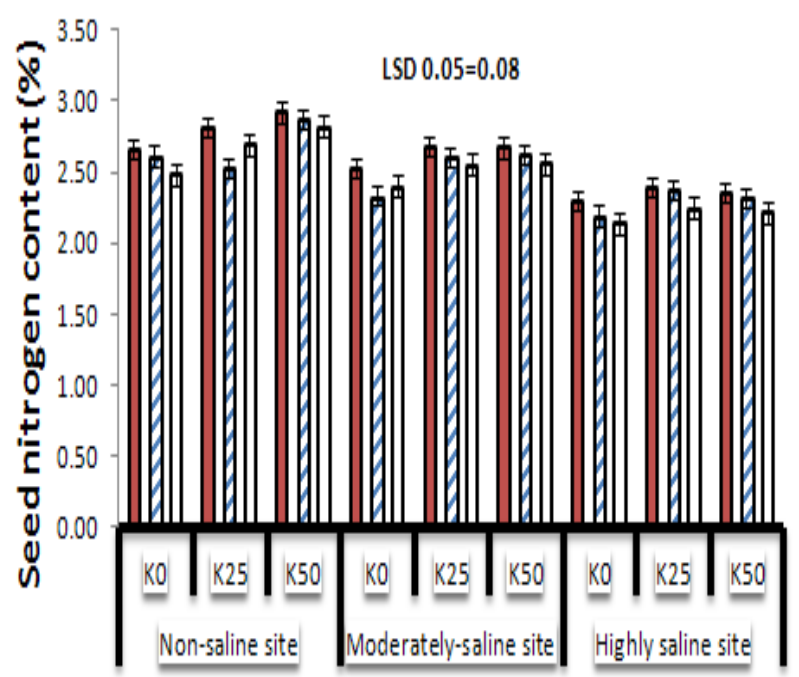

QShandaweel-3 QGiza 32 QSohag 1

j-Seed nitrogen content, \% 


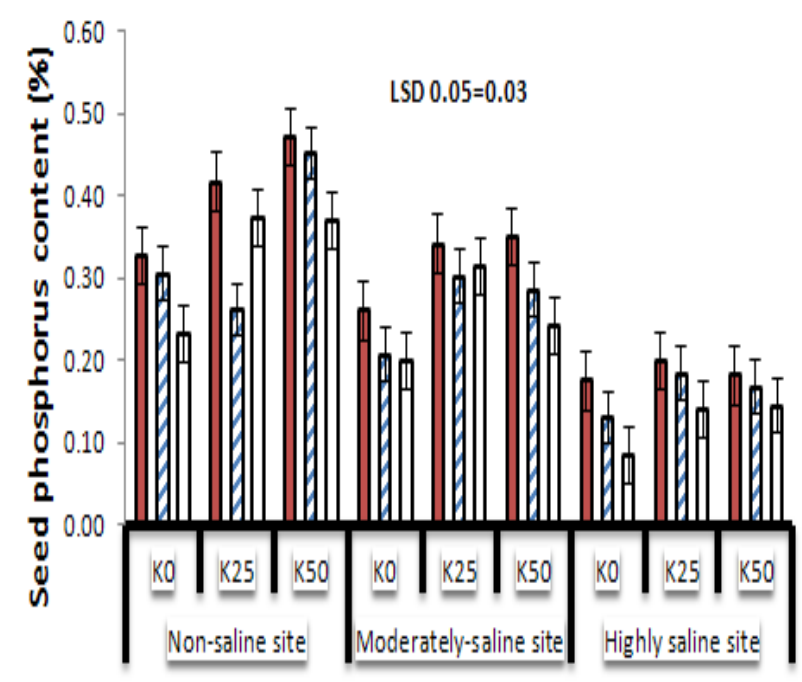

QShandaweel-3 QGiza 32 QSohag 1

k-Seed phosphorus content, \%

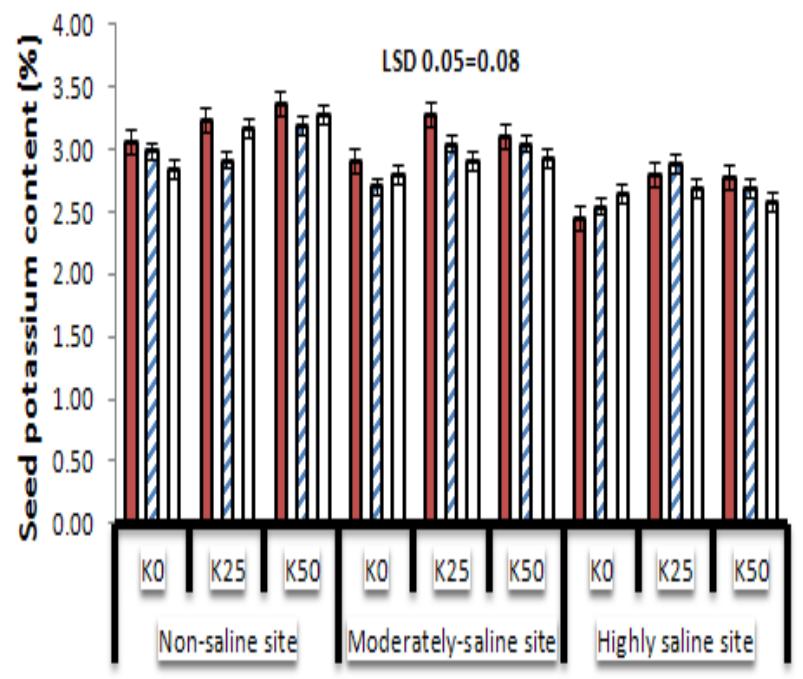

QShandaweel-3 QGiza 32 QSohag 1

I-Seed potassium content, $\%$

Figure 1. Effect of triple interaction among sites, potassium fertilizer levels and sesame varieties on all studied traits

\section{Conclusion}

From the previous results, we concluded that salt-affected soil limited yield of evaluated varieties. Potassium fertilizer had a vital role in improving the tolerance of the evaluated varieties under saline stress conditions. The yielding capacity of Shandaweel 3 under saline stress conditions was superior with compared to other varieties. Moreover, the preference was in favor of sowing Shandaweel 3 under saltaffected soil, with application of potassium fertilizer at a high rate $50 \mathrm{~kg} \mathrm{~K}_{2} \mathrm{O}$ per feddan for most studied traits.

\section{References}

A.O.A.C. (1975). 'Official Methods of Analysis "Association Official Analytical Chemists', $10^{\text {th }}$ Ed., Washington, D.C., USA.

A.O.A.C. (1995). 'Official Methods of Analysis of the Association Official Analytical Chemists', $15^{\text {th }}$ Ed., Washington, D.C., USA.

Abdel-Rahman, A.H. (2014). 'Effect of mineral potassium, compost and biofertilizers on soil physio-chemical properties and productivity of sesame grown on salt affected soils', Journal of Soil Sciences and Agricultural Engineering, 5 (6), pp. 791805.

Ahmad, F., Ahmad, J., Nawaz, H., Abbas, M.W., Shah, M.A., Iqbal, S., Mehmood, Z., and Ali, M. (2018). 'Influence of sulphur and potassium levels on yield and yield attribute of sesame (Sesamum indicum L.)', Research in Agriculture Livestock and Fisheries, 5 (2), pp. 147-150.

Ali, M.A., Tariqul, I.M., and Islam, M.T. (2005). 'Effect of salinity on some morphophysiological characters and yield in three sesame cultivars', Journal of the Bangladesh Agricultural University, 3 (2), pp. 209-214.

Desingh, R., and Kanagaraj, G. (2020). 'Effect of salinity stress on photosynthetic enzymes of two sesame (Sesamum indicum L.) varieties', Plant Archives, 20 (1), 16-20.

Elmasry, M.M., Huda, (2009). 'Effect of organic, inorganic and biofertilizers on growth, yield and physiological activities of 
soybean crop', M.Sc. Thesis, Fac. of Sci., Sohag Univ.

Gaballah, M.S., Abou, B., Leila, El-Zeiny, H.A., and Khalil, S. (2007). 'Estimating the performance of salt stressed sesame plant treated with antitranspirants', Journal of Applied Sciences Research, 3 (9), pp. 811817.

Gomez, K.A., and Gomez, A.A. (1984). 'Statistical Procedures for Agricultural Research', $2^{\text {nd }}$ Ed. New York: John Willey and Sons Inc.

Hafiz, S.I., and El-Bramawy, M.A.S. (2012). 'Response of sesame (Sesamum indicum L.) to phosphorus fertilization and spraying with potassium in newly reclaimed sandy soils', International Journal of Agricultural Science Research, 1 (3), pp. 34-40.

Jackson, M.L. (1958). 'Soil Chemical analysis'. Constable and Co. Ltd. London.

Jackson, M.L. (1967). 'Soil Chemical analysis'. Prentice-Hall, Inc., Englewood Cliffs, New Jersey, USA.

Jackson, M.L. (1973). 'Soil chemical analysis'. New Delhi: Prentice Hall of India Pvt. Ltd.

Jat, R., Naga, S.R., Choudhary, R., Jat, S., and Rolaniya, M.K. (2017). 'Effect of potassium and sulphur on growth, yield attributes and yield of sesame (Sesamum indicum L.)', Chemical Science Review and Letters, 6 (21), pp. 184-186.

Marschner, H. (1995). 'Mineral nutrition in higher plants'. (2 ${ }^{\text {nd }}$ Ed.), P. 861, Academic press, London, New York

Marschner, H. (2010). 'Mineral nutrition of higher plants'. Academic, London.

Nóbrega, J.S., Lopes, K.P., Santos, J.B.D., Paiva, F.J.D. S., Silva, J.G.D., and Lima, G.S.D. (2018). 'Quality of sesame seeds produced under soil salinity levels', Pesquisa
Agropecuária Tropical, 48 (3), pp. 280286.

Puvanitha, S., and Mahendran, S. (2017). 'Effect of salinity on plant height, shoot and root dry weight of selected rice cultivars', Scholars Journal of Agriculture and Veterinary Sciences, 4 (4), pp. 126-131.

Rasul, G. A. M. (2010). 'Effect of Potassium fertilizer on growth and yield of corn plants in some soils at Sulaimani governorate'. Mesopotamia Journal of Agriculture, 38 (1), pp. 35-44.

Suassuna, J.F., Fernandes, P.D., Brito, M. E. B., Arriel, N.H.C., de Melo, A.S., and Fernandes, J.D. (2017). 'Tolerance to Salinity of Sesame Genotypes in Different Phenological Stages', American Journal of Plant Sciences, 8, pp. 1904-1920.

Vadaliya, B.M., Parmar, K.B., Ribadiya, T.R., Vekaria, L.C., and Davra, M.A. (2019). 'Effect of salinity on yield, yield attributing characters and quality of sesame (Sesamum indicum L.) varieties', International Journal of Chemical Studies, 7 (1), pp. 2278-2281. 